EPiC Series in Engineering
Volume 3, 2018, Pages 800-809
HIC 2018. 13th International
Conference on Hydroinformatics

\title{
Development of a modularity index for reliability assessment of isolation valve systems
}

\author{
Orazio Giustolisi ${ }^{1 *}$ \\ ${ }^{1}$ Politecnico di Bari, Via E. Orabona 4, 70125 Bari, Italy \\ orazio.giustolisiepoliba.it
}

\begin{abstract}
Mechanical reliability refers to the assessment of the capacity of the water distribution network (WDN) to provide a correct service to the different type of costumers under abnormal operating conditions due to a failure of a system component. It depends on the effectiveness of the isolation valve system (IVS) and on the failure probability of components. Starting from the calculation of the actual customer demands during abnormal operating conditions of the hydraulic systems due to valve shutdowns and the failure probability of the separated segments, the work develops a metric for WDN reliability assessment. The finding is that the topologic part of WDN reliability assessment, relating to the IVS, is based on the risk of disconnection. Starting from it, the works develops a special modularity index for IVS reliability assessment.
\end{abstract}

\section{Introduction}

Around the world, comprehensive asset management of water distribution networks (WDNs) is becoming a relevant issue for technical-scientific research because water companies ask for solutions to novel tasks. The research and technique around WDNs come from a technical tradition of hydraulic verification. I.e. the main need of constructing WDNs in order to deliver water increasing the percentage of customers reached by the service or for fire protection have conditioned theories and solutions of the technical research during the last century as well as the limited computational burden and amount of flow and pressure measurements.

Starting the new millennium, aging of the hydraulic systems built during the past century, demographic pressure in towns, increased sensibility of the customers to the service quality versus the tariffs, etc. are moving the technical needs towards an effective asset management. Then, the traditional pipe sizing or calibration of pipe resistance to verify the hydraulic capacity of the system for customers and fire protection are tasks inside the most general needs of building district metering areas (DMAs), planning rehabilitation, providing water quality and security, increasing system reliability, etc. Overall,

${ }^{*}$ Corresponding author: orazio.giustolisi@poliba.it 
the reported changes in the perception of WDN management, ask for rationale, replicable and flexible methodologies for analysing, planning and designing solutions for the novel tasks allowing the socioeconomic and financial sustainability for the community and for water companies.

In this framework of novel technical-scientific research needs, the information and communication technologies (ICT) and the increased computational burden today allow storing asset and hydraulic data in geographic information systems (GIS), analysing large size networks, designing optimal solutions for novel tasks, communicating and synchronizing management data with company information systems. Furthermore, in the last few years the concept of internet of things (IoT) together with the route for fast $5 \mathrm{G}$ networks are providing new perspectives for designing more and more efficient solutions inside a rationale asset management. However, although the opportunity of ICT, computational burden, IoT, $5 \mathrm{G}$ networks, big data and data-science cannot substitute the "physicallybased" studies on hydraulic systems, they should be obligatory considered in advanced modelling approaches to system analyses, planning and design of solutions for novel tasks in order to obtain the maximum efficiency and sustainability of WDN asset management.

Then, starting from the graph theory of the last century, advanced theories and approaches to the study of networks have been proposed in the last decade by several researchers (Albert \& Barabasi, 2002; Newman, 2010; Barthélemy, 2011 ). This novel field of science, named complex network theory (CNT), aiming at building a science of network analyses, assumes the paradigm that the majority of the systems work as networks. WDNs are a special case of infrastructure spatial networks (Watts \& Strogatz, 1998), i.e. they are material network whose construction is constrained by the physical factors as buildings, streets and terrain conditions as opposite to immaterial networks as for example Internet.

CNT allows studying the connectivity the network feature (Watts \& Strogatz, 1998; Barabási \& Albert, 1999; Lämmer, et al., 2006), performing division in modules/segments (clustering) (Newman \& Girvan, 2004; Newman, 2006; Newman, 2006; Fortunato \& Barthélemy, 2007; Fortunato, 2010), assessing system vulnerability (Holme, et al., 2002; Albert, et al., 2004; Latora \& Marchioni, 2005; Iyer, et al., 2013; Hajebi, et al., 2016; Campbell, et al., 2016; Zhang, et al., 2017), etc. CNT have been already used for WDN division in modules/segments (Perelman \& Ostfeld, 2011; Scibetta, et al., 2013; Diao, et al., 2013; Di Nardo, et al., 2014; Giustolisi \& Ridolfi, 2014) being commonly conceived for planning DMAs. Inside this context, some researchers have recently proposed tailored metrics for WDN segmentation originating from the modularity index (Newman \& Girvan, 2004) considering conceptual cuts close to the ending nodes instead of in the middle of pipes (Giustolisi \& Ridolfi, 2014; Giustolisi, et al., 2015) in order to account for the actual installation of devices to build DMAs. Using the same concepts, the dual division of the network considering nodes was recently performed by Simone et al. (Simone, et al., 2016) for sampling design in order to identify topological districts having pressure meters at the boundary nodes, which is related to line graph and overlapping communities. Those are few examples of using tailored CNT studies in order to rationalize and innovate two technical tasks related to the recent perspective of availability of a bigger amount of data from flow and pressure measurements.

The present work aims at building general indicators for reliability assessment, which are based on hydraulic behaviour and connectivity features (topology) of the WDN considering that the two aspects are correlated because the network topology influences the hydraulics being its domain.

The term reliability generally refers to the ability of the system to provide adequate performance for customers considering abnormal operating conditions (Xu \& Goulter, 1999). It is usually studied according to two general classes of failure events (Farmani, et al., 2005): mechanical and hydraulic failures. The first refers to the system components failure, such as pipe breaks and pumps out of service, whereas the second accounts for the variability of demands and/or pipe hydraulic resistances. Reliability for WDNs has been widely analysed over the years. For example, Todini (Todini, 2000) developed the resilience index as a measure of WDN performance under failure conditions based on the power required at each node. Later, Prasad and Park (Prasad \& Park, 2004) modified the original Todini's resilience index (Todini, 2000) by accounting for the uniformity in diameter of pipes connected at each 
node as a surrogate measure of the redundancy of water paths in the system in case of mechanical failure and recently Creaco et al. (Creaco, et al., 2016) extended Todini's index (Todini, 2000) to the pressuredriven analysis. The reported indices considered the alteration of water supply service with respect to normal operating conditions in face of limited alteration of network topology.

Actually, a consistent classification of the reliability with respect to the technical reality needs to consider not only the type of failure event but also the occurrence of valve shutdowns to separate the section/segment of the network where planned or unplanned works occur (Walski, 1993; Walski, 1993; Jun \& Loganathan, 2007; Giustolisi \& Savic, 2000; Alvisi, et al., 2011). In fact, a significant burst event can be seen as a hydraulic failure considering the pressure drop due to the increase flow into the WDN or a mechanical failure with respect to the need of repairing it after separating a section of the hydraulic system.

From the hydraulic modelling standpoint, Tanyimboh et al. (Tanyimboh, et al., 2011) addressed the need of computing reliability indices based on a variant of classical demand-driven analysis (DDA) because of the WDN possible incapability to deliver the full service to customers during abnormal operating conditions. Today, pressure-driven analysis (PDA) is recognized providing more realistic picture of the hydraulic system behaviour during pressure deficient conditions than DDA (Giustolisi \& Walski, 2012). Pressure deficient conditions typically occur in the still connected network after valve shutdowns because the topology connectivity of the hydraulic system might result significantly modified. Also, significant burst flows, the increasing of customer consumption (e.g. in touristic areas) with respect to the hydraulic capacity, failure of pumping systems and pressure reduction valves, etc. might cause pressure deficient conditions to be examined with PDA. However, the occurrence of valve shutdowns altering the domain of the hydraulics of the system should mandatory ask for analyses considering such topological changes of the network.

The aim of this work is the development a WDN reliability assessment indicator based on the actually supplied customer demand. Therefore, PDA will be the basis of the indicator development and consistently with the above premise three contributions to the reliability are evidenced: (i) the hydraulic behaviour of the still connected network; (ii) the topological performance of the IVS and (iii) the topological performance of IVS with respect to unintended isolation (Walski, 1993) (Walski, 1993).

The finding is that the topological part of the proposed WDN reliability assessment indicator allows assessing the performance of the IVS. Such performance is measured by the risk of disconnection, i.e. the probability of segment failure per the required customer demand over time. Finally, a modularity index for IVS performance assessment is developed.

\section{Mechanical reliability assessment indicators}

Starting from the hydraulic modelling, the present section develops the nodal reliability indicators for the actual supplied customer demand, which allow assessing the spatial system reliability over time. Then, the proposed nodal indicator allows defining the overall WDN reliability assessment indicator over time and of the operative cycle. The definition of reliability with respect to customer demands could be extended to fire demands, although the contemporarily occurrence of a fire during planned or unplanned works is statistically irrelevant.

\subsection{Hydraulic model}

A hydraulic network of $n_{p}$ pipes with unknown flow rates/discharges, $n_{n}$ nodes with unknown heads (internal nodes) and $\mathrm{n} 0$ nodes with known heads (tank levels, for example) can be analysed by solving the following system based on energy and mass balance equations as for example in the comprehensive work by Giustolisi and Walski (Giustolisi \& Walski, 2012), 


$$
\begin{aligned}
& \mathbf{A}_{p p}(t) \mathbf{Q}_{p}(t)+\mathbf{A}_{p n} \mathbf{H}_{n}(t)=-\mathbf{A}_{p 0} \mathbf{H}_{0}(t) \\
& \mathbf{A}_{n p} \mathbf{Q}_{p}(t)-\mathbf{d}_{n}(t)\left(\mathbf{H}_{n}(t)\right)=\mathbf{0}_{n}
\end{aligned}
$$

where $\mathbf{Q}_{p}$ is the column vector of unknown pipe flow rates; $\mathbf{H}_{n}$ is the column vector of unknown nodal heads; $\mathbf{H}_{0}$ is the column vector of known nodal heads and $\mathbf{d}_{n}$ is the column vector of demands, which depends on Hn. $\mathbf{A}_{\mathrm{pn}} \mathbf{A}_{\mathrm{np}}$ and $\mathbf{A}_{\mathrm{p} 0}$ are topological incidence sub-matrices of general topological matrix, link-node, of the network. The subscript $\mathrm{p}$ and $\mathrm{n}$ indicate the dependence of the matrices and vectors on the number of pipes and nodes (related to unknown heads), while the subscript " 0 " refers to the number of reservoirs (known heads). The model of Eqs. (1) simulates the snapshot in " $t$ " of the hydraulic system behaviour over time e.g. with the demands but also other boundary conditions such as tank levels, pressure reduction valve status, etc. In fact, the reliability indicators should refer to an operative cycle of the hydraulic network and, therefore, extended period simulation (EPS) is mandatory. For example, $\mathbf{d}_{n}\left(\mathbf{H}_{n}\right)=\mathbf{d}_{n}{ }^{a c t}\left(\mathbf{H}_{n}\right)+\mathbf{d}_{n}{ }^{l}\left(\mathbf{H}_{n}\right)$ where $\mathbf{d}_{n}{ }^{a c t}$ and $\mathbf{d}_{n}{ }^{l}=\left[n_{n}, 1\right]$ column vectors of demands related to customers (Wagner, et al., 1988) and to background leakage flow (Giustolisi \& Walski, 2012), respectively.

Hydraulic modelling of Eqs. (1) refers to PDA, i.e. to the assumption of pressure-dependent leakages and the capability to simulate the nodal condition of pressure falling below the value for a sufficient/correct service, i.e. the pressure deficient conditions for customer demands (Tanyimboh, et al., 2011; Giustolisi \& Walski, 2012). PDA is mandatory for mechanical reliability analysis because the hydraulic system component failure might cause a significant pressure deficient condition for customer demands, while the classic DDA cannot approximate PDA in such situation (Tanyimboh, et al., 2011; Giustolisi \& Walski, 2012).

\subsection{Reliability indicators for actual supplied customer demand and nodal pressure}

We here develop reliability indicators assuming the intervention for maintenance asking for valve shutdowns of the IVS. To this purpose, we define the fraction of nodal customer demand. The fraction is computed with respect to the normal condition over time for each maintenance event, i.e. valve shutdowns. The PDA in EPS allows computing such fraction, whose formulation is

$$
f_{d}(i, s, t)=\frac{d^{a c t}(i, s, t)}{d^{r e q}(i, t)} \quad \forall i \in n_{n} \quad \forall s \in n_{s} \quad \forall t \in[1, T]
$$

where $f_{d}$ is the fraction of customer demand; $d^{a c t}$ is the actual customer demand computed in PDA using the Wagner's model (Giustolisi \& Walski, 2012) (note that for nodes of separated portion of the hydraulic system due to valve shutdowns $d^{a c t}$ is null and the fraction $f_{d}$ is null); $d^{r e q}$ is the "statistical" required customer demand varying over time depending on the demand patterns (Giustolisi \& Walski, 2012); $i$ and $t$ are subscripts indicating the $i$ th node and the time $t$ of the EPS over the operative cycle $T ; s$ is a subscript indicating the failure event into the $s$ th segment of the IVS; $n_{n}$ and $n_{s}$ are the number of nodes and segments, respectively. The actually supplied demand during a valve shutdown event corresponding to each of the $n_{s}$ scenarios of segment separation refers to two situations: $i$ th node belongs (a) or not (b) to the separated portion of the network.

In the case (a), the actually supplied customer demand and nodal pressure are null for $i$ th node, i.e. $f_{d}(i \in S, s, t)$ is null. This case should not be confused with the case of unintended isolations (Walski, 1993; Walski, 1993; Jun \& Loganathan, 2007; Giustolisi \& Savic, 2000) corresponding to possible disconnection of a node from source(s) of water although it does not belong to the separated portion of the network. 
In the case (b), $f_{d}(i, s, t)$ ranges in $[0,1]$ with null value corresponding to totally unsupplied customer demand and unitary value corresponding to correct service.

After computing the fractions of Eq. (2), in order to obtain an overall indicator of reliability for each $i$ th node over time $t$, we need to weigh those values over the $n_{s}$ scenarios of segment isolation because the corresponding probability of occurrence is different.

Let assume that the normalized probability of a failure event in the $s$ th segment is $\Pi(s)$, the reliability indicators becomes:

$$
R I^{f d}(i, t)=\sum_{s=1}^{n_{s}} \Pi(s) f_{d}(i, s, t)=\frac{\sum_{s=1}^{n_{s}} \Pi(s) d^{a c t}(i, s, t)}{d^{r e q}(i, t)} \quad \forall i \in n_{n} \quad \forall t \in[1, T](3)
$$

where $R I^{f d}$ is the reliability indicator for the customer demand for each $i$ th node and over time $t$. The reliability indicator of Eq. (3) is not fully informative about the reason of a reliability value. We mean that the value of the reliability is determined by system hydraulic capacity because the valve shutdowns for separating the $s$ th segment can cause a pressure deficient condition at the $i$ th node although $i \notin s$, and by the topology of IVS because the supplied demand at the $i$ th node is null every time $i \in s$. Therefore, we define

$$
R I H^{f d}(i, t)=\Pi(s) \delta\left(\mathrm{S}_{s}, \mathrm{~S}_{i}\right)+R I^{f d}(i, t)
$$

where $R I H^{f d}$ is the hydraulic reliability indicator for customer demand for each $i$ th node and over time $t$. Kronecker's $\delta$ is unitary if the $i$ th node belongs to the $s$ th segment $\left(i \in s \Leftrightarrow \mathrm{S}_{s}=\mathrm{S}_{i}\right)$ otherwise is null. It worth to note that $R I H$ is nothing than $R I$ plus the probability of disconnection $\Pi(s)$ for $i \in s$. Moreover, $R I H^{f d}$ is unitary for $d^{a c t}(i, s, t)=d^{r e q}(i, t)$ because the summation of $\Pi(s)$ is unitary by definition. It means that if the network design is robust with respect to each sth segment disconnection the pressure deficient condition does not occur in the still connected nodes and the reliability depends on the IVS only while $R I H^{f d}$ is unitary. This fact demonstrates that the mechanical reliability $\left(R f^{f d}\right)$ depends on topology by the IVS and on hydraulic behaviour. Therefore, the actual IVS is relevant for assessing reliability and cannot surrogated by indicators, which do not consider the actual topology changes in the network.

In order to clarify Eq. (4), we here assume that the IVS separates the WDN in two segments and that the normalized probabilities of a failure event are $\Pi(s=1)=0.3$ and $\Pi(s=2)=0.7$, hence,

$$
\begin{array}{ll}
R I^{f d}(i, t)=\frac{\Pi(2) d^{a c t}(i, 2, t)}{d^{\text {req }}(i, t)}=\frac{0.7 d^{a c t}(i, 2, t)}{d^{\text {req }}(i, t)} & \\
\operatorname{RIH}^{f d}(i, t)=\Pi(1)+\frac{\Pi(2) d^{a c t}(i, 2, t)}{d^{\text {req }}(i, t)}+=0.3+\frac{0.7 d^{\text {act }}(i, 2, t)}{d^{\text {req }}(i, t)} & \\
\operatorname{RI}^{f d}(i, t)=\frac{\Pi(1) d^{a c t}(i, 1, t)}{d^{\text {req }}(i, t)}=\frac{0.3 d^{\text {act }}(i, 1, t)}{d^{\text {req }}(i, t)} & \\
\operatorname{RIH}^{f d}(i, t)=\Pi(2)+\frac{\Pi(1) d^{a c t}(i, 1, t)}{d^{\text {req }}(i, t)}=0.7+\frac{0.3 d^{a c t}(i, 2, t)}{d^{\text {req }}(i, t)} & \forall i \in s=2
\end{array}
$$


Eqs. (5) show that the reliability indicator related to customer demand ranges in [0,0.7] for nodes belonging to segment $s=1$ and in $[0,0.3]$ for nodes of segment $s=2$. Therefore, assuming $d^{\text {act }}(i, 2, t)=$ $d^{r e q}(i, t)$ in the second of Eqs. (5) $R I H^{f d}$ is unitary and, from the first of Eqs. (5), the upper bound of the reliability is 0.7 for nodes belonging to $s=1$. For the same reasoning the upper bound of the reliability is 0.3 for nodes belonging to $s=2$.

Consequently, we can state that a topological part of the demand reliability indicator influences its upper bounds and we can write:

$$
T I^{f d}(s, i)=\Pi(s) \delta\left(\mathrm{S}_{s}, \mathrm{~S}_{i}\right) \Leftrightarrow T I^{f d}(s, i)=R I H^{f d}(i, t)-R I^{f d}(i, t)
$$

The definition of the topological indicators $T f^{f d}$ allows a better understanding the role of the IVS in the reliability of the hydraulic system. The definition of $R I H^{f d}$ needs to be expanded in order to consider that unintended isolations which are also a topological issue of the IVS,

$$
R I H^{f d}(i, t)=\Pi(s) \delta\left(\mathrm{S}_{s}, \mathrm{~S}_{i}\right)+\sum_{s=1}^{n_{s}}\left[\Pi(s)\left(\frac{d^{a c t}(i, s, t)}{d^{r e q}(i, t)}\right)^{i \in c o n}+(\Pi(s))^{i \notin c o n}\right]\left(1-\delta\left(\mathrm{S}_{s}, \mathrm{~S}_{i}\right)\right)
$$

where inside the summation appear two terms relating to the following occurrences: (a) $i$ th node is

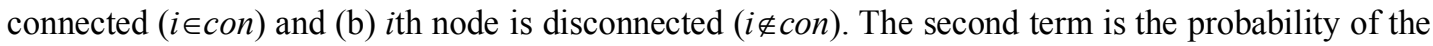
sth segment, to which $i$ does not belong, because of the factor $\left(1-\delta\left(\mathrm{S}_{s}, \mathrm{~S}_{i}\right)\right)$. Then it is the probability of unintentional disconnection of the $i$ th node (Walski, 1993; Walski, 1993; Jun \& Loganathan, 2007) when a portion of the network is isolated by means of valve shutdowns for maintenance works. Therefore,

$$
\begin{aligned}
& U I^{f d}(i, s)=\sum_{s=1}^{n_{s}}(\Pi(s))^{i \notin c o n}\left(1-\delta\left(\mathrm{S}_{s}, \mathrm{~S}_{i}\right)\right) \\
& \Leftrightarrow \operatorname{RIH}^{f d}(i, t)=R I^{f d}(i, t)+T I^{f d}(s, i)+U I^{f d}(s, i)
\end{aligned}
$$

where $U f^{d}$ is the topological indicator related to the unintended isolations and it is under a summation because the $i$ th node can be unintentionally disconnected for more than one different valve shutdowns event.

\section{Reliability indicator of the hydraulic system}

Considering the Eq. (3), the reliability indicator of the entire hydraulic network, $R R^{\text {met }}(t)$, can be defined as follows,

$$
R I^{n e t}(t)=\frac{\sum_{i=1}^{n_{n}} \sum_{s=1}^{n_{s}} \Pi(s) d^{a c t}(i, s, t)}{D(t)=\sum_{i=1}^{n_{n}} d^{r e q}(i, t)} \quad \forall t \in[1, T]
$$

and from the Eq. (4), it is possible to write, 


$$
\begin{aligned}
& R I^{n e t}(t)=R I H^{n e t}(t)-T I^{n e t}(t) \\
& T I^{n e t}(t)=\sum_{i=1}^{n_{n}} \sum_{s=1}^{n_{s}} \Pi(s)\left(\boldsymbol{\rho}_{n}(t)\right)_{i} \delta\left(\mathrm{S}_{s}, \mathrm{~S}_{i}\right)=\sum_{s=1}^{n_{s}} \Pi(s) \sum_{i=1}^{n_{i}}\left(\boldsymbol{\rho}_{n}(t)\right)_{i} \delta\left(\mathrm{S}_{s}, \mathrm{~S}_{i}\right)
\end{aligned}
$$

where $\boldsymbol{\rho}_{n}(t)$ is the vector of the $i$ th required demand normalized to the total demand in $t$ and $T T^{\text {net }}$ is topological indicator of the network similarly to Eq. (6). $T I^{\text {net }}$ formulation can be given also as

$$
T I^{n e t}(t)=\sum_{s=1}^{n_{s}} \Pi(s) \Delta(s, t)=\sum_{s=1}^{n_{s}}\left(\sum_{k=1}^{n_{p}}\left(\boldsymbol{\pi}_{p}\right)_{k} \delta\left(\mathrm{S}_{s}, \mathrm{~S}_{k}\right) \sum_{i=1}^{n_{i}}\left(\boldsymbol{\rho}_{n}(t)\right)_{i} \delta\left(\mathrm{S}_{s}, \mathrm{~S}_{i}\right)\right)
$$

where the $\Delta(s, t)$ is the summation of the element in $\boldsymbol{\rho}_{n}(t)$ belonging to $s$ th segment. To account for unintended isolation, following a similar reasoning, we could obtain the part accounting for the unintended isolations. Finally, a different way to write Eq. (11), expanded with unintended, is

$$
R I^{n e t}(t)=R I H^{n e t}(t)-\sum_{s=1}^{n_{s}} R_{d}(s, t)-\sum_{s=1}^{n_{s}} R_{u}(s, t)
$$

where $R_{d}$ and $R_{u}$ are the risk of disconnection and the risk of unintended isolations relating to the $s$ th segment. They are the product of the probability of failure of the sth segment and the damage computed as the inability to supply the required customer demand at time $t$. It is worth noting that Eq. (12) states that the IVS assessment depends on the risk of disconnection, unintended isolations comprised.

\section{Modularity index for IVS}

The modularity index (Newman \& Girvan, 2004) WDN-oriented (Giustolisi \& Ridolfi, 2014) is the basis of the optimal segmentation. Its formulation is,

$$
Q\left(\mathbf{w}_{p}\right)=\left\{1-\frac{n_{c}}{n_{p}}\right\}-\left\{\sum_{m=1}^{n_{m}}\left[\sum_{k=1}^{n_{p}} \frac{\left(\mathbf{w}_{p}\right)_{k} \delta\left(\mathrm{M}_{m}, \mathrm{M}_{k}\right)}{W}\right]^{2}\right\}=Q_{1}-Q_{2}
$$

where $n_{c}$ is number of "conceptual cuts" in the network (i.e. the decision variables of the segmentation problem); $n_{m}$ is the number of modules $\mathrm{M}$ and $\mathbf{w}_{p}$ is the column vector of pipe weights whose sum is $W$. Noting that the original formulation names modules the segments, we can write

$$
Q\left(\boldsymbol{\omega}_{p}\right)=\left\{1-\frac{n_{c}}{n_{p}}\right\}-\left\{\sum_{s=1}^{n_{s}} \Omega(s)^{2}\right\}=Q_{1}-Q_{2}
$$

Where $\omega_{p}$ is the column vector of the normalized pipe weights and $\Omega(s)$ is their summation for the sth segments. It is worth to note that the term $Q_{1}$ of Eqs. (13) and (14) decreases with the number of cuts, while $Q_{2}$ generally decreases with the number of modules/segments and, for a given number of modules/segments, it decreases with the similarity of modules to each other with respect to the assumed pipe weights. Therefore, $T T^{\text {net }}$ of Eq. (11) is similar to the term $Q_{2}$ of Eq. (14), but in the case of $T I^{\text {net }}$ the 
summation of $\Pi(s)$ and $\Delta(s, t)$ are unitary while in the case of $Q_{2}$ the summation of $\omega(s)$ is unitary. We can state that $T T^{\text {net }}$ is composed by the product of distinct normalized variables while $Q_{2}$ by the product of a single normalized variable per itself (power two).

Then, we can extend the concept of modularity index of Eq. (14)

$$
Q\left(R_{d}\right)=\left\{1-\frac{n_{c}}{n_{p}}\right\}-\left\{\sum_{s=1}^{n_{s}} \Pi(s) \Delta(s, t)\right\}=\left\{1-\frac{n_{c}}{n_{p}}\right\}-\left\{\sum_{s=1}^{n_{s}} R_{d}(s, t)\right\}=Q_{1}-Q_{2}
$$

Where $Q\left(R_{d}\right)$ is a modularity index minimizing the risk of disconnection with similar properties with the classic modularity index tailored for WDN (Giustolisi \& Ridolfi, 2014). Therefore, we can minimize the number of isolation valves to be installed in the $n_{c}$ "conceptual cuts" versus the maximization of the modularity index for IVS of Eq. (15) in order to obtain the optimal tradeoff between number of valves and minimization of $T I^{\text {net }}$, generally meaning maximization of the reliability $R I^{\text {net }}$ for a given number of isolation valves. A further formulation of Eq. (15) is

$$
Q_{\mathrm{IVS}}\left(\boldsymbol{\pi}_{p}, \boldsymbol{\rho}_{p}\right)=\left\{1-\frac{n_{c}}{n_{p}}\right\}-\left\{\sum_{s=1}^{n_{s}}\left(\sum_{k=1}^{n_{p}}\left(\boldsymbol{\pi}_{p}\right)_{k} \delta\left(\mathrm{S}_{s}, \mathrm{~S}_{k}\right) \sum_{i=1}^{n_{i}}\left(\boldsymbol{\rho}_{n}(t)\right)_{i} \delta\left(\mathrm{S}_{s}, \mathrm{~S}_{i}\right)\right)\right\}=Q_{1}-Q_{2-\mathrm{IVS}}
$$

where $Q_{\text {IVS }}$ is the modularity index for IVS, which better recalls the classic formulation of Eq. (13) while $Q_{2 \text {-IVS }}$ is the term of the metric characterizing the $Q_{\mathrm{IVS}}$ with respect to the classic modularity $Q$. However, the formulation of $Q_{\text {Ivs }}$ in Eq. (16) suffers of a resolution limit (Fortunato \& Barthélemy, 2007; Giustolisi \& Ridolfi, 2014), namely that increasing the number of cuts and the network size $n_{c}$ $Q_{1}$ tends to prevail on $Q_{2}$. In other words, it is not possible to identify small segments/modules because adding a single cut to separate a new one cannot not reduce enough, theoretically demonstrable as in Giustolisi and Ridolfi (Giustolisi \& Ridolfi, 2014), $Q_{1}$ with respect to $Q_{2}$. Therefore, in order to increase the resolution limit, it is possible to define the infrastructure modularity index for IVS as follows,

$$
I Q_{\mathrm{IVS}}\left(\boldsymbol{\pi}_{p}, \boldsymbol{\rho}_{p}\right)=\left\{1-\frac{n_{c}}{n_{p}}+\frac{n_{s}-1}{n_{p}}\right\}-\left\{\sum_{s=1}^{n_{s}}\left(\sum_{k=1}^{n_{p}}\left(\boldsymbol{\pi}_{p}\right)_{k} \delta\left(\mathrm{S}_{s}, \mathrm{~S}_{k}\right) \sum_{i=1}^{n_{i}}\left(\boldsymbol{\rho}_{n}(t)\right)_{i} \delta\left(\mathrm{S}_{s}, \mathrm{~S}_{i}\right)\right)\right\}=I Q_{1}-Q_{2-\mathrm{IVS}}
$$

where $I Q_{\text {IVS }}$ is the new metrics and $I Q_{1}$ is the new accounting for the number of segments $n_{s}$. For further details, the reader should refer to Giustolisi and Ridolfi (Giustolisi \& Ridolfi, 2014).

\section{Final remarks}

The present work presented the development a network reliability indicator with respect to actually supplied customer demand. Three contributions to the reliability are extrapolated: (i) the hydraulic behavior of the still connected network; (ii) the topological performance of the IVS and (iii) the topological performance of IVS with respect to unintended isolation (Walski, 1993; Walski, 1993). The finding is that the topological performance of the IVS depends on the risk of disconnection, i.e. the probability of segment failure per the required customer demand over time. Furthermore, CNT allowed rationalizing the finding and introducing the modularity index for IVS. This is a clear example that a bridge exists between hydraulic and CNT studies being both based on the same network domain. 
Finally, the author argues that in the next years CNT will allow rationalizing WDN studies. This fact will allow designing replicable methodologies and flexible solutions to the novel, different and variable tasks of the modern asset management of WDNs.

\section{Reference}

Albert, R., Albert, I. \& Nakarado, G., 2004. Structural vulnerability of the North American power grid. Phys. Rev. E, 69(025103).

Albert, R. \& Barabasi, A., 2002. Statistical mechanics of complex networks. Rev. Mod. Phys., 74(1), pp. 47-97.

Alvisi, S., Creaco, E. \& Franchini, M., 2011. Segment identification in water distribution systems. Urban Water, 8(4), pp. 203-217.

Barabási , A. \& Albert, R., 1999. Emergence of scaling in random networks. Science, Issue 286, p. 509-11.

Barthélemy, M., 2011 . Spatial networks. Physics Reports, 499(1-3), pp. 1-101.

Campbell, E. et al., 2016. A flexible methodology to sectorize water supply networks based on social network theory concepts and multiobjective optimization. J. Hydroinf., 18(1), pp. 62-76.

Creaco, E., Franchini, M. \& Todini, E., 2016. Generalized resilience and failure indices for use with pressure driven modeling and leakage. J. Water Res. Plan. Manage., 142(8).

Di Nardo, A. et al., 2014. Water Network Sectorization Based on Graph Theory and Energy Performance Indices. J. Water Resour. Plann. Manage., 140(5), pp. 620-629.

Diao, K., Zhou, Y. \& W., R., 2013. Automated Creation of District Metered Area Boundaries in Water Distribution Systems. J. Water Res. Plan. Manage., 139(2), pp. 184-190.

Farmani, R., Walters, G. \& Savic, D., 2005. Trade-off between total cost and reliability for anytown water distribution network. J. Water Resour. Plann. Manage., 131(3), pp. 161-171.

Fortunato, S., 2010. Community detection in graphs. Phys. Rep., 486(3-5), pp. 75-104.

Fortunato, S. \& Barthélemy, M., 2007. Resolution limit in community detection. Proc. Nat. Acad. Sciences of the USA, Volume 104, pp. 36-41.

Giustolisi, O. \& Ridolfi, L., 2014. A new modularity-based approach to segmentation of water distribution networks. J. Hydr. Eng., 140(10), pp. 1-14.

Giustolisi, O. \& Ridolfi, L., 2014. A novel infrastructure modularity index for the segmentation of water distribution networks. Water Resour. Res., 50(10), pp. 7648-7661.

Giustolisi, O., Ridolfi, L. \& Berardi, L., 2015. General metrics for segmenting infrastructure networks. J. Hydroinf., 17(4), pp. 505-517.

Giustolisi, O. \& Savic, D., 2000. Identification of segments and optimal isolation valve system design in water distribution networks. Urban Water J., 7(1), pp. 1-15.

Giustolisi, O. \& Walski, T., 2012. Demand components in water distribution network analysis. $J$. Water Resour. Plann. Manage., 138(4), pp. 356-367.

Hajebi, S. et al., 2016. Water distribution network sectorisation using graph theory and manyobjective optimization. J. Hydroinf., 18(1), pp. 77-95.

Holme, P., Kim, B. \& Yoon, C., 2002. Attack vulnerability of complex networks. Phys. Rev. E, 65(056109).

Iyer, S., Killingback, T., Sundaram, B. \& Wang, Z., 2013. Attack robustness and centrality of complex networks,. Plos One, 8(4).

Jun, H. \& Loganathan, G., 2007. Valve-Controlled Segments in Water Distribution Systems. J. Water Resour. Plann. Manage., 133(2), pp. 145-155.

Lämmer, S., Gehlsen, B. \& Helbing, D., 2006. Scaling laws in the spatial structure of urban road networks,. Physic A, 363(1), pp. 89-95. 
Latora, V. \& Marchioni, M., 2005. Vulnerability and protection of infrastructure networks. Phys. Rev. E, 71(015103).

Newman, M., 2006. Modularity and community structure in networks. Proc. Natl. Acad. Sci. U.S.A., 103(3), pp. 8577-8582.

Newman, M., 2006. Spectral methods for network community detection and graph partitioning. Phys. Rev. E, 88(042822).

Newman, M., 2010. Networks: An introduction. Oxford (UK).: Oxford Univ. Press.

Newman, M. \& Girvan, M., 2004. Finding and evaluating community structure in networks. Phys. Rev. E, 69(026113).

Perelman, L. \& Ostfeld, A., 2011. Topological clustering for water distribution systems analysis,. Environ. Modell. \& Software, 26(7), pp. 969-972.

Prasad, T. \& Park, N.-S., 2004. Multiobjective genetic algorithms for design of water distribution networks. J. Water Resour. Plann. Manage., 130(1), pp. 73-82.

Scibetta, M., Boano, F., Revelli, R. \& Ridolfi, L., 2013. Community detection as a tool for complex pipe network clustering. Europhysics Letters, 103(4).

Simone, A., Giustolisi, O. \& Laucelli, D., 2016. A proposal of optimal sampling design using a modularity strategy. Water Resour. Res., 52(8), pp. 6171-6185.

Tanyimboh, T., Tabesh, M. \& Burrows, R., 2011. Appraisal of source head methods for calculating reliability of water distribution networks. J. Water Resour. Plann. Manage., 127(4), pp. 206-213.

Todini, E., 2000. Looped water distribution networks design using a resilience index based heuristic approach,. Urban Water J., 2(2), pp. 115-122.

Wagner, J., Shamir, U. \& Marks, D., 1988. Water distribution reliability: simulation methods. $J$. Water Res. Plan. and Manage., 114(3), pp. 276-294.

Walski, T., 1993. Practical aspects of providing reliability in water distribution systems,. Reliab. Eng. Syst. Safety, 42(1), pp. 13-19.

Walski, T., 1993. Water distribution valve topology for reliability analysis. Reliab. Eng. Syst. Safety, 42(1), pp. 21-27.

Watts, D. \& Strogatz, D., 1998. Collective dynamics of small-world networks. Nature, p. 393-440..

Xu, C. \& Goulter, I., 1999. Reliability-based optimal design of water distribution network,. J. Water Resour. Plann. Manage., 125(6), pp. 352-362.

Zhang, Q. et al., 2017. Automatic Partitioning of Water Distribution Networks Using Multiscale Community Detection and Multiobjective Optimization. J. Water Resour. Plann. Manage., 143(9). 